\title{
Editorial
}

\section{Development and plasticity of multisensory functions}

\author{
Brigitte Röder ${ }^{\mathrm{a}, *}$ and Mark Wallace ${ }^{\mathrm{b}}$ \\ ${ }^{a}$ Biological Psychology and Neuropsychology, University of Hamburg, Hamburg, Germany \\ ${ }^{\mathrm{b}}$ Vanderbilt Brain Institute, Vanderbilt University, Nashville, TN, USA
}

\section{Introduction}

Most events in the world are specified by information from multiple senses. To take advantage of this redundant and complementary sensory information, the brain has specialized circuits that integrate these cues in order to shape perception and guide behavior. Because of the importance of these processes, it should come as no surprise that the field of multisensory research is expanding at a rapid rate (Driver and Noesselt, 2008; Stein and Stanford, 2008). Although basic questions about multisensory circuits and the encoding processes within them are beginning to be revealed, substantially less is known about how multisensory functions emerge during development (Lewkowicz and Lickliter, 1994) and how mutable these processes are across the life span (Bavelier and Neville, 2002). The present special issue "Development and plasticity of multisensory functions" addresses each of these topics and provides significant insights into the state-of-the-field for multisensory research. Both review and research articles report recent results of studies using both human and animal models, and which employ either prospective or retrospective approaches. Contributions focus on the major themes of multisensory development in children, cross-modal plasticity in the blind and deaf, and recovery of multisensory function following the restoration of vision or hearing. The following narrative provides a brief synopsis of the major research highlights contained within this special issue.

\footnotetext{
${ }^{*}$ Corresponding author. E-mail: brigitte.roeder@uni-hamburg.
}

The contribution from Grossman reviews recent behavioural and electrophysiological studies on the development of emotion perception during infancy, with an emphasis on the integration of the emotional content of visual (i.e., face) and auditory (i.e., voice) signals. In their contribution, Sathian and Stilla review evidence for the role of visual cortex in supporting nonvisual (i.e., tactile and auditory) functions in both sighted and blind individuals. Supporting evidence is provided by Amedi, Raz, Malach and Zohary, who report brain imaging results showing that tactile object recognition recruits ventral stream visual areas (i.e., the lateral occipital complex) both in sighted and blind humans. Complementing this is the contribution from Fiehler and Rösler, which focuses on dorsal visual stream functions such as space perception and action control, and suggests that these functions emerge in the absence of vision and are strongly shaped by inputs from nonvisual systems.

Shifting to deficits in auditory function, Dye and Bavelier review the evidence for visual attentional abilities in deaf adults, and attempt to provide a unified framework for reconciling the conflicting observations of both improved and degraded attentional function in the deaf. Botari, Nava, Ley and Pavani report new data on visual detection and discrimination performance in deaf compared to hearing adults, and argue that the advantages that are seen can reflect plasticity in sensory representations rather than changes in attentional networks.

Two contributions focus on the plasticity engendered by the restoration of vision after prolonged deprivation. Royal, Krueger, Fister and Wallace report on the re- 
sults of experiments in dark-reared animals and which show that early sensory experience is a prerequisite for the emergence of normal function in multisensory circuits. Consistent with these findings, Putzar, Hötting and Röder show that patients born with dense bilateral cataracts that were later removed are impaired not only in face recognition but also in integrating auditory and visual speech signals.

The final theme focuses on the restoration of hearing via cochlear implantation and ensuing changes in multisensory function. The contribution of the Peterson, Pisoni and Miyamoto deals with audiovisual speech perception and discusses outcome predictors for success post-implantation. In a related contribution, Tremblay, Champoux, Leopore and Theoret indexed multisensory function in cochlear implant (CI) recipients using the McGurk effect, an audiovisual illusion created by the perceptual fusion of discordant auditory and visual speech cues. They found remarkable similarities in the fusion abilities of normal hearing individuals and "proficient" CI users, both of whom appeared to show a greater reliance on the auditory channel. In contrast, "nonproficient" CI users appeared to rely more on the visual signal. Bergeson, Houston and Miyamoto compared congenitally deaf individuals fitted with a cochlear implant within the first year of life with normal hearing infants in using an intermodal preferential looking paradigm as a measure of audiovisual speech processing. They found that deaf children with CIs gradually developed the ability to match the auditory and visual cues, suggesting a period of early plasticity in which these associations can be established. Finally, Gilley, Sharma, Mitchell and Dorman used a non-speech task (redundant stimulus paradigm) to examine multisensory development, and found that multisensory-mediated performance gains were comparable between normal hearing and early implanted patients, but not for the late implanted.

Taken together, the results reviewed and reported in this special issue highlight the recent advances in our understanding of normal human multisensory development, as well as provide a wealth of evidence for the remarkable neural and perceptual plasticity that can accompany loss of function in one sensory system. It is our hope that this issue will spur both interest and future investigation in this fertile area of research, and which will enrich our knowledge about the development and plasticity of multisensory systems.

\section{References}

Amedi, A., Raz, N., Azulay, H., Malach, R. \& Zohary E. (2010). Cortical activity during tactile exploration of objects in blind and sighted humans. Restor Neurol Neuro, 28, 43-52.

Bavelier, D., \& Neville, H.J. (2002). Cross-modal plasticity: where and how? Nat Rev Neurosci, 3, 443-52.

Bergeson, T.R., Houston, D.M. \& Miyamoto, R.T. (2010). Effects of Congenital Hearing Loss and Chochlear Implantation an Audiovisual Speech Perception in Inflants and Children. Restor Neurol Neuro, 28, 157-165.

Bottari, D., Nava, E., Ley, P. \& Pavani, F. (2010). Enhanced reactivity to visual stimuli in deaf individuals. Restor Neurol Neuro, 28, 167-179.

Driver, J., \& Noesselt, T. (2010). Multisensory interplay reveals crossmodal influences on 'sensory-specific' brain regions, neural responses, and judgments. Neuron, 57(1), 11-23.

Dye, M.W.G. \& Bavelier, D. (2010). Attentional enhancements and deficits in deaf populations: An integrative review. Restor Neurol Neuro, 28, 181-192.

Fiehler, K. \& Rösler, F. (2010). Plasticity of multisensory dorsal stream functions: Evidence from congenitally blind and sighted adults. Restor Neurol Neuro, 28, 193-205.

Gilley, P.M., Sharma, A., Mitchell, T. \& Dorman, M.F. (2010). The influence of a sensitive period for auditory-visual integration in children with chochlear implants. Restor Neurol Neuro, 28, 207-218.

Grossmann, T. (2010). The development of emotion perception in face and voice during infancy. Restor Neurol Neuro, 28, 219-236.

Lewkowicz, D. \& Lickliter, R. (1994). The development of intersensory perception. Hillsdale, NJ: Lawrence Erlbaum Associates, Publishers.

Peterson, N.R., Pisoni, D.B. \& Miyamoto, R., T. (2010). Cochlear Implants and Spoken Language Processing Abilities: Review and Assessment of the Literature. Restor Neurol Neuro, 28, 237-250.

Putzar, L., Hötting, K. \& Röder, B. (2010). Early visual deprivation affects the development of face recognition and of audio-visual speech perception. Restor Neurol Neuro, 28, 251-257.

Royal, D.W., Krueger, J., Fister, M.C. \& Wallace, M.T. (2010). Adult plasticità of spatiotemporal receptive fields. Restor $\mathrm{Neu}$ rol Neuro, 28, 259-270.

Sathian, K. \& Stilla, R. (2010). Cross-modal Plasticity of tactile Perception in blindness. Restor Neurol Neuro, 28, 271-281.

Stein, B.E., \& Stanford, T.R. (2010). Multisensory integration: current issues from the perspective of the single neuron. Nat Rev Neurosci, 9(4), 255-66.

Tremblay, C., Champoux, F., Lepore, F. \& Theóret, H. (2010). Audiovisual fusion and chochlear implant proficiency. Restor Neurol Neuro, 28, 283-291. 